\title{
MIXED MOTIVES AND ALGEBRAIC CYCLES, I
}

\author{
MASAKI HANAMURA
}

The idea of motives goes back to A. Grothendieck (cf. [Kl] [Ma]). As he worked on the Weil conjecture on the zeta function of a variety $X$ over a finite field $k$, he was in need of studying the actions of the Frobenius maps on the $\ell$-adic etale cohomologies $H^{i}\left(X \otimes_{k} \bar{k}, \mathbb{Q}_{\ell}\right)$ for different $\ell$; to prove the conjecture one must show that the characteristic polynomial is a $\mathbb{Q}$-polynomial independent of $\ell$, and its eigenvalues have expected absolute values. He postulated certain conjectures on algebraic cycle classes, called the standard conjectures, and observed that they imply the Weil conjecture.

The standard conjectures are concerned not only with $\ell$-adic cohomology, but with any other Weil cohomology (such as Betti or de Rham cohomology) as well, and the consequences are further-reaching. They indicate the existence of the theory of (pure Grothendieck) motives : there is a semisimple abelian category of Grothendieck motives $\mathcal{M}(k)$ (where $k$ is any ground field) satisfying the properties:

(1) To a smooth projective variety $X$ over $k$, its motive $h(X)$, an object of $\mathcal{M}(k)$, is associated; $h(X)$ is contravariantly functorial in $X$.

(2) Any Weil cohomology $H^{*}$ (which is a contravariant functor from the category of smooth projective varieties to the category of graded vector spaces) factors through $\mathcal{M}(k)$. The resulting functor (called the realization functor) from $\mathcal{M}(k)$ to the space of graded vector spaces, is exact and faithful.

Morally, the vector space $H^{*}(X)$ together with its functorial behavior is ruled by the motive $h(X)$. The functor $h$ is the universal Weil cohomology taking values in a semi-simple abelian category.

In view of the discovery of mixed Hodge structures by Deligne - the theory of weights for Betti cohomology [De 2], one may naturally suspect the existence of the category of mixed motives - motives with weights, which contains the "motives" of quasi-projective singular varieties. This abelian category is no longer semi-simple, which makes it all the more interesting, because extension groups are non-trivial. The significance of

Received September 13, 1995.

The author was partially supported by the NSF. 
such a category was made clear by the conjecture that these extension groups should be related to Chow groups and higher $K$-groups of varieties, $[\mathrm{Be}],[\mathrm{De}]$; see also [Ja].

The purpose of this note is to outline the construction of the triangulated category $\mathcal{D}(k)$ of mixed motives (over a field $k$ ) which has the expected properties. Such a construction was made possible incorporating the theory of higher Chow groups due to S. Bloch. We perceive a mixed motive as a diagram built up of smooth projective varieties and correspondences (in the sense of higher Chow groups) between them. The full account of the materials here will appear elsewhere.

We would like to warmly thank S. Bloch for his collaboration on this project. For other approaches to categorical constructions, see [Le 2].

\section{Preliminaries}

Higher Chow groups [Bl 1]. Throughout this paper $k$ is a fixed ground field.

Let $\square^{n}=\mathbb{A}_{k}^{n}$ with coordinates $\left(x_{1}, \cdots, x_{n}\right)$. Faces of $\square^{n}$ are intersections of codimension one faces, and the latter are divisors of the form $\square_{i, a}^{n-1}=\left\{x_{i}=a\right\}$ where $a=0$ or 1 . A face of dimension $m$ is canonically isomorphic to $\square^{m}$. Let $\Sigma_{n}$ be the permutation group of the set $\{1, \cdots, n\}$ acting on $\square^{n}$ by permutations of the coordinates.

Let $X$ be an equi-dimensional variety (or a scheme) over a field $k$. Let $\mathcal{Z}^{r}(X, n)$ be the $\mathbb{Q}$-vector space of $\mathbb{Q}$-cycles $z$ of codimension $r$ on $X \times \square^{n}$ such that

(i) each irreducible component of $z$ meets each face $X \times \square^{n}$ properly, and

(ii) $z$ is alternating with respect to $\Sigma_{n}$, namely, for any $\sigma \in \Sigma_{n}, \sigma(z)=$ $\operatorname{sgn}(\sigma) z$. The inclusions of codimension one faces $\delta_{i, a}: \square_{i, a}^{n-1} \hookrightarrow \square^{n}$ induce the map

$$
\partial=\sum(-1)^{i+a} \delta_{i, a}^{*}: \mathcal{Z}^{r}(X, n) \rightarrow \mathcal{Z}^{r}(X, n-1)
$$

(namely, $\partial$ sends alternating cycles to alternating ones) and $\left(\mathcal{Z}^{r}(X, \cdot), \partial\right)$ is a homology complex. We call this the cycle complex (of codimension $r$ ) of $X$. By definition the (rational) higher Chow groups are the homology groups of this complex:

$$
C H^{r}(X, n)=H_{n} \mathcal{Z}^{r}(X, \cdot) \text {. }
$$

Note $C H^{r}(X, 0)=C H^{r}(X)$, the rational Chow group of $X$.

In [Bl-Le], considering $\mathbb{Z}$ - cycles on $X \times \square^{n}$ which are "normalized", in place of $\mathbb{Q}$-cycles, integral higher Chow group is defined, and denoted by 
$C H^{r}(X, n)$. In this paper we only consider rational cycle complexes and rational Chow groups. Rational Chow groups are isomorphic to Adams graded pieces of $K$-groups of $X$.

Theorem 1.1 [Bl 2] [Le 1]. There is a canonical isomorphism

$$
C H^{r}(X, n) \cong K_{n}^{\prime(r)}(X)_{\mathbb{Q}}
$$

where $K_{n}^{\prime}(X)$ is the $K$-group of the exact category of coherent sheaves on $X, K_{n}^{\prime}(X)_{\mathbb{Q}}=K_{n}^{\prime}(X) \otimes \mathbb{Q}$, and $K_{n}^{\prime(r)}(X)_{\mathbb{Q}}$ is the $r$-th Adams eigenspace.

Grothendieck's construction [Ma]. For an equi-dimensional smooth projective variety $X$ over $k$, let $C^{r}(X)$ be the $\mathbb{Q}$-vector space of $\mathbb{Q}$-algebraic cycles of codimension $r$ on $X$ modulo an adequate equivalence relation. An element of $C^{*}(X \times Y)$ is called a $C$-correspondence from $X$ to $Y$. The composition of correspondences is the map

$$
C^{\operatorname{dim} X+a}(X \times Y) \otimes C^{\operatorname{dim} Y+b}(Y \times Z) \rightarrow C^{\operatorname{dim} X+a+b}(X \times Z)
$$

which sends $u \otimes v$ to

$$
v \circ u=\left(p_{X Z}\right)_{*}\left(p_{X Y}^{*} u \cdot p_{Y Z}^{*} v\right) .
$$

Here $p_{X Y}$, for example, is the projection $X \times Y \times Z \rightarrow X \times Y$.

By definition, the category $C \mathcal{M}(k)$ of $C$-motives has objects of the form $(X, P)(r)$ where $r \in \mathbb{Z}, X$ is a not necessarily connected smooth projective variety, and $P=\left(P_{i}\right) \in \oplus_{i} C^{\operatorname{dim} X_{i}}\left(X_{i} \times X_{i}\right)$ where $X=\coprod X_{i}, X_{i}$ equidimensional, $\operatorname{dim} X_{i} \neq \operatorname{dim} X_{j}$ if $i \neq j$, and $P \circ P=P$. The homomorphism group is given by

$$
\operatorname{Hom}((X, P)(r),(Y, Q)(s))=\bigoplus_{i} Q \circ C^{\operatorname{dim} X_{i}+s-r}\left(X_{i} \times Y\right) \circ P_{i}
$$

the composition is induced from the composition of correspondences.

The object $(X, P)(r)$ is the image of the endomorphism of $X(r)$ given by $P$.

When $C^{*}$ is the rational Chow group (resp. algebraic cycles modulo numerical equivalence), the resulting category we denote by $C H \mathcal{M}(k)$ and call the category of Chow motives (resp. $\mathcal{M}(k)$, the category of Grothendieck motives ). 


\section{$\S$ 2. The category $\mathcal{D}(k)$}

We will define the three triangulated categories $\mathcal{D}(k), \mathcal{D}_{\text {finite }}(k)$, and $\mathcal{D}_{\text {inf }}(k)$. The category $\mathcal{D}(k)$ is called the triangulated category of mixed motives. We first construct the category $\mathcal{D}_{\text {finite }}(k)$ of finite diagrams and the larger category $\mathcal{D}_{\text {inf }}(k)$ of (possibly) infinite diagrams. Then we construct $\mathcal{D}(k)$ as an intermediate triangulated category:

$$
\mathcal{D}_{\text {finite }}(k) \hookrightarrow \mathcal{D}(k) \hookrightarrow \mathcal{D}_{\text {inf }}(k)
$$

(2.1). In this section, unless otherwise stated, $X, Y, \cdots$ will denote irreducible smooth projective varieties over $k$. We consider formal symbols of the form

$$
\bigoplus_{\alpha \in I} X_{\alpha}\left(r_{\alpha}\right)
$$

where $X_{\alpha}$ is a smooth projective variety, $I$ an index set (possibly infinite) and $r_{\alpha} \in \mathbb{Z}$. We call such a symbol infinite (resp. finite) if $I$ is infinite (resp. finite). We write 0 for the corresponding symbol when $I$ is an empty set.

Define dual of a finite symbol by:

$$
\left(\oplus X_{\alpha}\left(r_{\alpha}\right)\right)^{\vee}=\oplus X_{\alpha}\left(\operatorname{dim} X_{\alpha}-r_{\alpha}\right) .
$$

Tensor product of two (possibly infinite) symbols is defined by

$$
\left(\oplus X_{\alpha}\left(r_{\alpha}\right)\right) \otimes\left(\oplus X_{\alpha^{\prime}}^{\prime}\left(r_{\alpha^{\prime}}\right)\right)=\oplus\left(X_{\alpha} \times X_{\alpha^{\prime}}^{\prime}\right)\left(r_{\alpha}+r_{\alpha^{\prime}}^{\prime}\right) ;
$$

and, when the first object is finite, inner Hom by

$$
\underline{\operatorname{Hom}}\left(\oplus X_{\alpha}\left(r_{\alpha}\right), \oplus X_{\alpha^{\prime}}^{\prime}\left(r_{\alpha^{\prime}}\right)\right)=\left(\oplus X_{\alpha}\left(r_{\alpha}\right)\right)^{\vee} \otimes\left(\oplus X_{\alpha^{\prime}}^{\prime}\left(r_{\alpha^{\prime}}\right)\right) .
$$

Define the cycle complex of a formal symbol by

$$
\mathcal{Z}^{0}\left(\oplus X_{\alpha}\left(r_{\alpha}\right), \cdot\right)=\oplus \mathcal{Z}^{r_{\alpha}}\left(X_{\alpha}, \cdot\right) .
$$

This is a homological complex, but often regarded as a cohomological complex in the obvious manner. (If $C_{\bullet}$ is a homological complex, $C^{\bullet}=C_{-}$• is the corresponding cohomological complex.) Note there is a partially defined map

$$
\begin{gathered}
\mathcal{Z}^{0}\left(\underline{\operatorname{Hom}}\left(X_{1}\left(r_{1}\right), X_{2}\left(r_{2}\right)\right), \cdot\right) \otimes \mathcal{Z}^{0}\left(\underline{\operatorname{Hom}}\left(X_{2}\left(r_{2}\right), X_{3}\left(r_{3}\right)\right), \cdot\right) \\
--\rightarrow \mathcal{Z}^{0}\left(\underline{\operatorname{Hom}}\left(X_{1}\left(r_{1}\right), X_{3}\left(r_{3}\right)\right), \cdot\right)
\end{gathered}
$$


given by the composition of correspondences

$$
u \otimes v \mapsto v \circ u=p_{13 *}\left[\left(X_{1} \times v\right) \cdot\left(u \times X_{3}\right)\right] .
$$

We also define,

$$
\begin{aligned}
& \mathcal{Z}^{0}\left(\underline{\operatorname{Hom}}\left(\oplus X_{\alpha}\left(r_{\alpha}\right), \oplus X_{\beta}\left(r_{\beta}\right)\right), \cdot\right): \\
& =\prod_{\alpha} \bigoplus_{\beta} \mathcal{Z}^{0}\left(\underline{\operatorname{Hom}}\left(X_{\alpha}\left(r_{\alpha}\right), X_{\beta}\left(r_{\beta}\right)\right), \cdot\right) .
\end{aligned}
$$

It consists of elements $\left(f_{\alpha \beta}\right)$ satisfying the condition that for each $\alpha$, there are only a finite number of $\beta$ 's such that $f_{\alpha \beta}$ is non-zero. If $\oplus_{\alpha}$ is a finite direct sum, this coincides with the cycle complex of $\underline{\operatorname{Hom}}\left(\oplus X_{\alpha}\left(r_{\alpha}\right)\right.$, $\left.\oplus X_{\beta}\left(r_{\beta}\right)\right)$; but in general it is not the cycle complex of the object $\underline{\operatorname{Hom}}(-$, -). Partly to avoid confusions, we will denote the same complex by $\mathcal{Z}^{0}\left(\underline{\operatorname{Hom}}\left(\oplus X_{\alpha}\left(r_{\alpha}\right), \oplus X_{\beta}\left(r_{\beta}\right)\right)^{\bullet}(\right.$ cohomological notation $)$. There is a partially defined map:

$$
\begin{gathered}
\mathcal{Z}^{0}\left(\underline { \operatorname { H o m } } ( \oplus X _ { \alpha } ( r _ { \alpha } ) , \oplus X _ { \gamma } ( r _ { \gamma } ) ) \bullet \otimes \mathcal { Z } ^ { 0 } \left(\underline{\operatorname{Hom}}\left(\oplus X_{\gamma}\left(r_{\gamma}\right), \oplus X_{\beta}\left(r_{\beta}\right)\right)^{\bullet}\right.\right. \\
--\rightarrow \mathcal{Z}^{0}\left(\underline{\operatorname{Hom}}\left(\oplus X_{\alpha}\left(r_{\alpha}\right), \oplus X_{\beta}\left(r_{\beta}\right)\right)^{\bullet}\right.
\end{gathered}
$$

given by

$$
(g \circ f)_{\alpha \beta}=\sum_{\gamma} g_{\gamma \beta} \circ f_{\alpha \gamma} .
$$

(Note this is a finite sum.)

Definition 2.2. An object of $\mathcal{D}_{\text {inf }}(k)$ (resp. $\left.\mathcal{D}_{\text {finite }}(k)\right)$ is a set of data called an infinite diagram (resp. finite diagram)

$$
K=\left(K^{m}\right)=\left(K^{m} ; f^{m, n}\right)
$$

where

(i) For each integer $m, K^{m}=\oplus_{\alpha \in I(m)} X_{\alpha}\left(r_{\alpha}\right)$, a possibly infinite formal symbol (resp. finite symbol). We require: For all but finitely many $m$ 's, $K^{m}=0$

(ii) For $(m, n)$ with $m<n$, given $f^{m, n}=\left(f_{\alpha \beta}^{m, n}\right) \in \mathcal{Z}^{0}\left(\underline{\operatorname{Hom}}\left(K^{m}, K^{n}\right)\right.$, $n-m-1)$, which are subject to the condition: For $m<n$, one has

$$
(-1)^{n} \partial f^{m, n}+\sum_{m<\ell<n} f^{\ell, n} \circ f^{m, \ell}=0, .
$$


On the left side the compositions of the correspondences are required to be defined. Note that the condition in the case $n=m+1$ reads: $\partial f^{m, m+1}=0$.

The object with all $K^{m}=0$ is denoted by 0 . The object with $K^{0}=p t$, $K^{m}=0$ for $m \neq 0$ is denoted by $\mathbb{Q}$.

Let the Tate objects be $\mathbb{Q}(r):=(p t(r)$ placed in degree $-2 r)$ for $r \in \mathbb{Z}$.

One can extend the definition of the cycle complex to objects of $\mathcal{D}$ inf $(k)$ so that there is a complex

$$
\mathcal{Z}^{0}(K, \cdot)
$$

associated to any object $K$.

Before doing this, we need some preparations. For $f \in \mathcal{Z}^{r}(X \times Y, \ell)$, consider the partially defined map (of graded vector spaces)

$$
f_{*}: \mathcal{Z}^{r}(X, \cdot)--\rightarrow \mathcal{Z}^{r+\ell}(Y, \cdot+\ell), \quad f_{*}(z)=p_{Y *}(f \cdot(z \times Y)) .
$$

There is a quasi-isomorphic subcomplex of $\mathcal{Z}^{r}(X, \cdot)$ on which $f_{*}$ is defined. In fact, the following holds. For each smooth projective variety $X$ there is a collection of distinguished subcomplexes of $\mathcal{Z}^{r}(X, \cdot)$ satisfying:

(i) For a distinguished subcomplex $\mathcal{Z}^{r}(X, \cdot)^{\prime}$, the inclusion into $\mathcal{Z}^{r}(X, \cdot)$ is a quasi-isomorphism;

(ii) For any cycle $f \in \mathcal{Z}^{r}(X \times Y, \ell)$, there is a distinguished subcomplex $\mathcal{Z}^{r}(X, \cdot)^{\prime}$ on which $f_{*}$ is defined and induces a map $f_{*}: \mathcal{Z}^{r}(X, \cdot)^{\prime} \rightarrow$ $\mathcal{Z}^{r+\ell}(Y, \cdot+\ell)$

(iii) The intersection of a finite collection of distinguished subcomplexes is again distinguished.

Note also that one has

$$
(\partial f)_{*}=\partial \circ f_{*}+(-1)^{\ell-1} f_{*} \circ \partial
$$

on $\mathcal{Z}^{r}(X, \cdot)^{\prime}$.

To construct $\mathcal{Z}^{0}(K, \cdot)$, for each $m$ and $\alpha \in I(m)$, take a distinguished subcomplex $\mathcal{Z}^{0}\left(X_{\alpha}\left(r_{\alpha}\right), \cdot\right)^{\prime}$ so that each $f_{\alpha \beta}^{m, n}$ induces the map $f_{\alpha \beta *}^{m, n}$ : $\mathcal{Z}^{0}\left(X_{\alpha}\left(r_{\alpha}\right), \cdot\right)^{\prime} \rightarrow \mathcal{Z}^{0}\left(X_{\beta}\left(r_{\beta}\right), \cdot\right)^{\prime}$. We then let

$$
\mathcal{Z}^{0}\left(K^{m}, \cdot\right)^{\prime}:=\oplus_{\alpha} \mathcal{Z}^{0}\left(X_{\alpha}\left(r_{\alpha}\right), \cdot\right)^{\prime}
$$

and we have $f^{m, n} *: \mathcal{Z}^{0}\left(K^{m}, \cdot\right)^{\prime} \rightarrow \mathcal{Z}^{0}\left(K^{n}, \cdot+(n-m-1)\right)^{\prime}$. This is possible since there are only finitely many $\beta$ 's with non-zero $f_{\alpha \beta}^{m, n}$. We define $\mathcal{Z}^{0}(K, \cdot)$ to be the complex $(\mathbb{K}, d)$ with

$$
\mathbb{K}^{i}=\bigoplus_{j \geq i} \mathcal{Z}^{0}\left(K^{j}, j-i\right)^{\prime}
$$


and

$$
d^{i}=\sum_{j}\left((-1)^{j} \partial_{j}+\sum_{j<\ell} f_{*}^{j, \ell}\right) .
$$

The condition (2.2), (ii) implies that this is a complex. The result is unique up to canonical quasi-isomorphism.

Let $(K, f)$ and $(L, g)$ be objects in $\mathcal{D}_{\text {inf }}(k)$. The function cycle complex $\mathcal{Z}^{0}(\underline{\operatorname{Hom}}(K, L))^{\bullet}$ is defined as follows. Let $\mathcal{Z}^{0}\left(\underline{\operatorname{Hom}}\left(K^{m}, L^{m^{\prime}}\right), \cdot\right)^{\prime}$ be distinguished subcomplexes such that

For $u \in \mathcal{Z}^{0}\left(\underline{\operatorname{Hom}}\left(K^{m}, L^{m^{\prime}}\right), \cdot\right)^{\prime}, u \circ f^{n, m}$ is defined and $\in \mathcal{Z}^{0}\left(\underline{\operatorname{Hom}}\left(K^{\prime} L^{m^{\prime}}\right), \cdot\right)^{\prime}$; similarly for $g^{m^{\prime}, n^{\prime}} \circ u$.

The cohomological complex to be defined has the group of $N$-cochains

$$
\mathcal{Z}^{0}(\underline{\operatorname{Hom}}(K, L))^{N}=\bigoplus_{-m+m^{\prime}-p=N} \mathcal{Z}^{0}\left(\underline{\operatorname{Hom}}\left(K^{m}, L^{m^{\prime}}\right), p\right)^{\prime}
$$

The differential of this complex, which we denote by $D$, is the sum of the three kinds of maps:

$$
\begin{gathered}
(-1)^{p+m^{\prime}+n+1}\left(\circ f^{n, m}\right): \mathcal{Z}^{0}\left(\underline{\operatorname{Hom}}\left(K^{m}, L^{m^{\prime}}\right), p\right)^{\prime} \rightarrow \\
\mathcal{Z}^{0}\left(\underline{\operatorname{Hom}}\left(K^{n}, L^{m^{\prime}}\right), p+n-m-1\right)^{\prime}, \\
(-1)^{m^{\prime}+n^{\prime}}\left(g^{m^{\prime}, n^{\prime}} \circ\right): \mathcal{Z}^{0}\left(\underline{\operatorname{Hom}}\left(K^{m}, L^{m^{\prime}}\right), p\right)^{\prime} \rightarrow \\
\mathcal{Z}^{0}\left(\underline{\operatorname{Hom}}\left(K^{m}, L^{n^{\prime}}\right), p+n^{\prime}-m^{\prime}-1\right)^{\prime},
\end{gathered}
$$

and

$$
\begin{gathered}
(-1)^{m^{\prime}} \partial: \mathcal{Z}^{0}\left(\underline{\operatorname{Hom}}\left(K^{m}, L^{m^{\prime}}\right), \cdot\right)^{\prime} \rightarrow \\
\mathcal{Z}^{0}\left(\underline{\operatorname{Hom}}\left(K^{m}, L^{m^{\prime}}\right), \cdot-1\right)^{\prime} .
\end{gathered}
$$

One verifies that this is a complex; it is unique up to canonical quasiisomorphism.

Given three objects $K, L$ and $M$, the partially defined composition map

$$
\begin{gathered}
\mathcal{Z}^{0}(\underline{\operatorname{Hom}}(K, L))^{\bullet} \otimes \mathcal{Z}^{0}(\underline{\operatorname{Hom}}(L, M))^{\bullet}--\rightarrow \mathcal{Z}^{0}(\underline{\operatorname{Hom}}(K, M))^{\bullet} \\
u \otimes v \mapsto v \circ u ;(v \circ u)^{m, n}=\sum_{\ell \in \mathbb{Z}} v^{\ell, n} \circ u^{m, \ell}
\end{gathered}
$$

is associative and satisfies the Leibniz formula

$$
D(v \circ u)=D v \circ u+(-1)^{\operatorname{deg} v} v \circ D u
$$


where $\operatorname{deg} v$ is the total degree of $v$ in the cohomological complex. One can show that there is a quasi-isomorphic subcomplex, unique up to canonical quasi-isomorphism, of the tensor product

$$
\mathcal{Z}^{0}(\underline{\operatorname{Hom}}(K, L))^{\bullet} \otimes \mathcal{Z}^{0}(\underline{\operatorname{Hom}}(L, M))^{\bullet}
$$

on which the composition $u \otimes v \mapsto v \circ u \in \mathcal{Z}^{0}(\underline{\operatorname{Hom}}(K, M))^{\bullet}$ is defined.

By definition

$$
\operatorname{Hom}_{\mathcal{D}_{\text {inf }}(k)}(K, L)=H^{0} \mathcal{Z}^{0}(\underline{\operatorname{Hom}}(K, L))^{\bullet} .
$$

The composition of morphisms is induced from the composition of the function complexes. A morphism $u: K \rightarrow L$ is represented by $u^{m, n} \in$ $\mathcal{Z}^{0}\left(\operatorname{Hom}\left(K^{m}, L^{n}\right), n-m\right)^{\prime}$ (for a choice of a collection of distinguished subcomplexes) subject to the condition

$$
(-1)^{n} \partial u^{m, n}-\sum(-1)^{m+\ell} u^{\ell, n} \circ f^{m, \ell}+\sum(-1)^{\ell+n} g^{\ell, n} \circ u^{m, \ell}=0 .
$$

It defines the zero morphism if there exist $U^{m, n} \in \mathcal{Z}^{0}\left(\underline{\operatorname{Hom}}\left(K^{m}, L^{n}\right), n-\right.$ $m+1)^{\prime}$ such that

$$
u^{m, n}=(-1)^{n} \partial U^{m, n}+\sum(-1)^{m+\ell} U^{\ell, n} \circ f^{m, \ell}+\sum(-1)^{\ell+n} g^{\ell, n} \circ U^{m, \ell} .
$$

\section{Proposition 2.3.}

(1) The category $\mathcal{D}_{\text {inf }}(k)$ is an additive category.

(2) The association $K \mapsto \mathcal{Z}^{0}(K, \cdot)$ is a functor $\mathcal{D}$ inf $(k) \rightarrow D(\mathbb{Q})$, the latter the unbounded derived category of $\mathbb{Q}$-vector spaces.

(3) There is a contravariant functor

$$
h:(\text { smooth proj. } / k) \rightarrow \mathcal{D}_{\mathrm{inf}}(k)
$$

which assigns a smooth projective variety $X$ to the diagram ( $X$ placed in degree 0 ).

All the statements are valid if $\mathcal{D}_{\text {inf }}(k)$ is replaced with $\mathcal{D}_{\text {finite }}(k)$.

It can also be shown that on the category $\mathcal{D}_{\text {inf }}(k)$ there is the operation of tensor product; the category $\mathcal{D}_{\text {finite }}(k)$ has tensor product, dual, and inner Hom.

There is a natural structure of triangulated category on $\mathcal{D}_{\text {inf }}(k)$ (resp. $\left.\mathcal{D}_{\text {finite }}(k)\right)$. For example, the translation functor $K \mapsto K[1]$ is defined as follows. On objects $(K[1])^{m}=K^{m+1}$ and $(f[1])^{m, n}=-f^{m+1, n+1}$. For a morphism $u: K \rightarrow L$ represented by $\left(u^{m, n}\right), u[1]: K[1] \rightarrow L[1]$ is to be represented by $(u[1])^{m, n}=u^{m+1, n+1}$.

Let $K$ be an object of $\mathcal{D}_{\text {inf }}(k)$, and $p: K \rightarrow K$ a projector. It can be shown that the image of $p$ exists in $\mathcal{D}_{\text {inf }}(k)$; denote it by $[K, p]$. Even if $K$ is in $\mathcal{D}_{\text {finite }}(k),[K, p]$ may not be in $\mathcal{D}_{\text {finite }}(k)$. Now we may give 
Definition 2.4. Let $\mathcal{D}(k)$ be the full subcategory of $\mathcal{D}_{\inf }(k)$ consisting of objects of the form $[K, p]$ with $K$ an object of $\mathcal{D}_{\text {finite }}(k)$ and $p$ a projector. This is a triangulated subcategory.

The category $\mathcal{D}(k)$ is closed under the operations of duals, tensor products, and inner Hom's.

The category of Chow motives is fully embedded in $\mathcal{D}(k)$ : in fact let the functor be:

$$
(X, P)(r) \mapsto[h(X)(r), P]
$$

where the right hand side is the image of the projector $P: h(X)(r) \rightarrow$ $h(X)(r)$. The object $h(X)(r)$ is $h(X) \otimes \underline{\mathbb{Q}}(r)$ by definition, or equivalently, the object " $X(r)$ in degree $-2 r$ ". This correspondence of objects preserves the homomorphism groups.

Theorem 2.5. There is a fully faithful functor $C H \mathcal{M}(k) \rightarrow \mathcal{D}(k)$.

The following summarizes the main properties on $\mathcal{D}(k)$ which can be shown without assuming any conjectures. The property (1) has been explained; (3) is clear from the definitions and Theorem (1.1).

Theorem 2.6. The triangulated category $\mathcal{D}(k)$ has the following properties:

(1) $\mathcal{D}(k)$ has duals, tensor product, inner Hom's, the unit object $\underline{\mathbb{Q}}$, and the Tate objects $\mathbb{Q}(r)$;

$(2)($ ch $(k)=0)$ The contravariant functor $h:($ smooth proj./k $) \rightarrow \mathcal{D}(k)$ extends to the category of quasi-projective varieties; to each quasi-projective $X$ there is associated its (cohomological) motive $h(X)$, an object of $\mathcal{D}(k)$;

(3) If $X$ is smooth and projective, one has

$$
\operatorname{Hom}_{\mathcal{D}(k)}(\underline{\mathbb{Q}}, h(X)(r)[2 r-m])=K_{m}^{(r)}(X) \otimes \mathbb{Q} .
$$

Here the right side is an Adams-graded piece of the $K$-group of $X$.

\section{$\S 3$. The motivic $t$-structure}

We consider the following "standard" conjectures on cycle classes in a Weil cohomology, and on the Chow groups and $K$-groups of smooth projective varieties.

(1) Grothendieck's standard conjectures [Kl]. These are conjectures on the $\mathbb{Q}$-vector space of cycle classes in Weil cohomologies. We do not state the individual conjectures here. We only note: (1-1) is one of the conjectures, and (1-2) is a consequence of the conjectures.

(1-1) Homological equivalence (with respect to a Weil cohomology) coincides with numerical equivalence for algebraic cycles.

(1-2) The category $\mathcal{M}(k)$ of Grothendieck motives is semi-simple and abelian. 
(2) The filtration conjecture by Murre [Mu]. Let $X$ be an irreducible smooth projective variety over a field $k$, and $\pi^{i}(i=0,1, \cdots, 2 \operatorname{dim} X)$ be the Kunneth components of the diagonal (for a Weil cohomology).

(A) The $\left\{\pi^{i}\right\}$ lifts to an orthogonal set of projectors $\left\{\Pi^{i}\right\}$ in $C H^{\operatorname{dim} X}$ $(X \times X)$ such that $\sum \Pi^{i}=\Delta_{X}$.

(B) The correspondences $\Pi^{2 r+1}, \cdots, \Pi^{2 \operatorname{dim} X}$ act as zero on $C H^{r}(X)$.

(C) For each $\nu \geq 0$, let $F^{\nu} C H^{r}(X)=\operatorname{Ker} \Pi_{*}^{2 r} \cap \operatorname{Ker} \Pi_{*}^{2 r-1} \cap \cdots \cap$ $\operatorname{Ker} \Pi_{*}^{2 r-\nu+1}$. Then $F^{\nu} C H^{r}(X)$ is independent of the liftings $\Pi^{i}$.

(D) $F^{1} C H^{r}(X)=C H^{r}(X)_{\text {hom }}$, where the latter is the subspace of classes of cycles homologically equivalent to zero ( for a Weil cohomology).

(3) Vanishing/Injectivity conjectures. Let $(X, P)$ be an object on $C H \mathcal{M}(k)$. One may speak of cohomological degrees with respect to a Weil cohomology.

a) (Vanishing) Suppose $(X, P)$ is of cohomological degrees $\geq 2 r-n$ if $n>0$ and $>2 r$ if $n=0$. Then one has

$$
P_{*} C H^{r}(X, n)=0 .
$$

b) (Injectivity) If $(X, P)$ is of pure cohomological degree $2 r$, then the cycle class map

$$
P_{*} C H^{r}(X) \rightarrow H^{2 r}(X)
$$

is injective.

Remark. In the case $P=\Delta_{X}$ the former conjecture is nothing but the vanishing conjecture of Soule-Beilinson. In the case $P=\Pi^{2 r}$, the latter conjecture is equivalent to Murre's conjecture (D).

Theorem 3.1. Assume the three conjectures (1) - (3) above. Then there is a t-structure on $\mathcal{D}(k)$ which is compatible with $H^{*}$ (= any of the de Rham, etale or Betti cohomology).

As a consequence, there is a particular abelian subcategory of $\mathcal{D}(k)$ - the heart of the $t$-structure. (For generalities on $t$-structure, see [BBD].) We propose this to be the abelian category of mixed motives.

\section{References}

[Be] A. Beilinson, Height pairing between algebraic cycles, Contemp. Math. Amer. Math. Soc. 67 (1987), 1-24.

[BBD] A. Beilinson, J. Bernstein, P. Deligne, Faisceaux Pervers, Asterisque 100 (1982), Société Mathématique de France.

[Bl 1] S. Bloch, Algebraic cycles and higher K-theory, Adv. in Math 61 (1986), 267304. 
[Bl 2] - The moving lemma for higer Chow groups, J. Alg. Geom. 3 (1994), 537-368.

[Bl-Le] S. Boch, M. Levine, Book in preparation.

[De] P. Deligne, A quois serve les motifs?, Proc. of Symp. in Pure Math. 55, 143-161.

[De 2] _ Theorie de Hodge II, Publ. Math. IHES 40 (1972), 5-57; Publ. Math. IHES 44 (1975), 6-77.

[Ja] U. Jannsen, Motivic sheaves and filtrations on Chow groups, Proc of Symp. in Pure Math. 55, 245-302.

[Kl] S. Kleiman, Algebraic cycles and Weil conjectures, Dix exposés sur la cohomologie des scémas, North Holland, Amsterdam, 1968, pp. 359-386.

[Le 1] M. Levine, Bloch's higer Chow groups revisited, preprint.

[Le 2] , The derives motivic category, preprint.

[Ma] Y. Manin, Correspondences, motifs and monoidal transformations, Math. USSR Sb. (1968), 439-470.

$[\mathrm{Mu}]$ J. Murre, On a conjectural filtration on the Chow groups of algebraic varieties, I, II, Indag Math. 4 (1968), 177-188; ibid, 189-201.

M Ax-Planck-Institüt für M Athematik, 53225 Bonn, Germany

E-mail address: hanamura@mpim-bonn.mpg.de 